\title{
Quantum-dot infrared photodetectors: a review
}

\author{
Adrienne D. Stiff-Roberts \\ Department of Electrical and Computer Engineering, Duke University, Durham, North \\ Carolina 27708-0291, USA \\ adrienne.stiffroberts@,duke.edu
}

\begin{abstract}
Quantum-dot infrared photodetectors (QDIPs) are positioned to become an important technology in the field of infrared (IR) detection, particularly for high-temperature, low-cost, high-yield detector arrays required for military applications. High-operating temperature $(\geq 150 \mathrm{~K})$ photodetectors reduce the cost of IR imaging systems by enabling cryogenic dewars and Stirling cooling systems to be replaced by thermo-electric coolers. QDIPs are well-suited for detecting mid-IR light at elevated temperatures, an application that could prove to be the next commercial market for quantum dots. While quantum dot epitaxial growth and intraband absorption of IR radiation are well established, quantum dot nonuniformity remains as a significant challenge. Nonetheless, state-of-the-art mid-IR detection at $150 \mathrm{~K}$ has been demonstrated using 70-layer InAs/GaAs QDIPs, and QDIP focal plane arrays are approaching performance comparable to $\mathrm{HgCdTe}$ at $77 \mathrm{~K}$. By addressing critical challenges inherent to epitaxial QD material systems (e.g., controlling dopant incorporation), exploring alternative QD systems (e.g., colloidal QDs), and using bandgap engineering to reduce dark current and enhance multi-spectral detection (e.g. resonant tunneling QDIPs), the performance and applicability of QDIPs will continue to improve.
\end{abstract}

Keywords: InAs/GaAs quantum dots, colloidal quantum dots, quantum-dot infrared photodetectors, QDIP focal plane arrays.

\section{INTRODUCTION}

Infrared (IR) photodetectors are used in a range of imaging applications, including military signature analysis, environmental monitoring, medical diagnosis, and industrial equipment diagnosis. The typical components of an IR camera system include: optics for light collection, a focal plane array (FPA) for detection and signal processing, a cooling system for photodetector arrays, and electronics for the digital images. A significant reduction in the cost, size, and weight of IR camera systems is possible if the traditional cooling systems, such as liquid-nitrogen dewars or Stirling coolers, are replaced by thermo-electric coolers. Such a design change requires the development of an IR photodetector that operates at elevated temperatures $(\geq 150 \mathrm{~K})$. Quantum-dot infrared photodetectors (QDIPs) have great potential to provide the requisite detector array performance at higher temperatures.

QDIPs use an extrinsic detection scheme in which the absorbed photon energy is less than the bandgap due to intraband photoexcitation enabled by three-dimensional quantum confinement of the photodetector active region. Through lithographical, epitaxial, or chemical techniques, active region heterostructures with dimensionality on the order of the deBroglie wavelength for an electron can be designed. Compared to higher-dimensional active regions, such as $\mathrm{AlGaAs} / \mathrm{GaAs}$ quantum wells (one-dimensional quantum confinement) and $\mathrm{HgCdTe}$ low-bandgap semiconductors (bulk material), important advantages of quantum dots for IR photodetection include inherent sensitivity to normal-incidence light, low dark current, and higher operating temperatures $(>150 \mathrm{~K})$. In addition, QDs provide many parameters for tuning the IR response, such as QD size and shape, strain, and material composition.

Since the first extensive QDIP characterization in 1999 at the University of Michigan [1], several research groups around the world [2-16] have investigated these devices, seeking to 
push the boundaries of state-of-the-art performance, particularly in the mid-IR wavelength range [17]. Low dark current [11, 18, 19], multi-spectral response [16, 20, 21], highdetectivity [22], high-temperature photodetection [23, 24], and IR imaging [25-28] have been demonstrated in QDIPs.

\section{THE STANDARD INAS/GAAS QDIP}

\subsection{Overview of device operation}

InAs quantum dots are embedded in a wider-bandgap matrix material (GaAs) by epitaxial overgrowth. Depending on the band-lineup of the QD and matrix materials, QD confinement barriers are created in the conduction and/or valence bands, thereby providing a mechanism for quantum confinement in addition to the nanoscale QD size. These confinement barriers are important in that, when combined with selective carrier occupation through doping, the independent control of electron and/or hole populations is enabled. A typical InAs/GaAs QD conduction band diagram under flat-band conditions is shown in Fig. 1. The QDIPs are unipolar devices and use electrons for charge transport in the InAs/GaAs material system. Free electrons are provided by doping (typically $\mathrm{Si}$ ) to occupy bound states of the QD. The standard InAs/GaAs QDIP device heterostructure, shown in Fig. 2, comprises a QD active region with top and bottom doped n-type contact layers. The QD active region typically comprises 10-30 InAs QD layers separated by large GaAs barriers to prevent the propagation of strain through the heterostructure. Device fabrication techniques include standard photolithography, metallization, and wet-etching to process two-terminal devices in a vertical configuration. When IR photons impinge on the QDIP, photo-excited carriers escape from the QD so that they can be collected as photocurrent. Once photoexcited carriers are in the continuum, they can experience several additional processes, such as: (i) drift in the continuum under the influence of an electric field, (ii) capture into the excited state of either the same QD or a different one, and (iii) collection at a device contact.

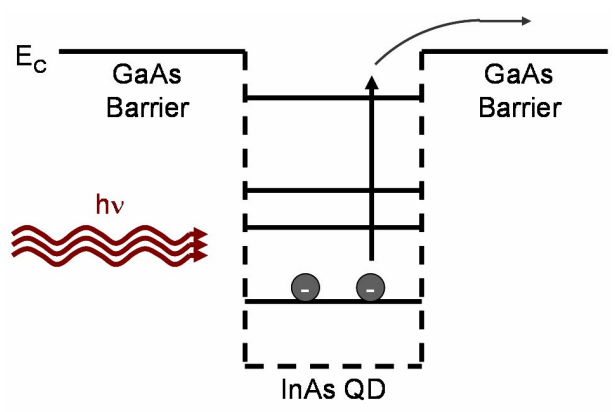

Fig. 1. Schematic diagram of conduction band (energy vs. position) demonstrating photocurrent generation in epitaxial, InAs/GaAs QDIPs. 


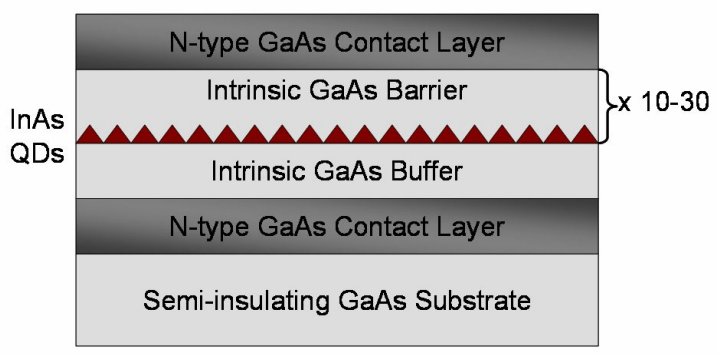

Fig. 2. Schematic diagram of device heterostructure of epitaxial, InAs/GaAs QDIPs.

\subsection{Strained-layer epitaxy of Stranski-Krastanow InAs/GaAs quantum dots}

Molecular beam epitaxy (MBE) routinely enables excellent control over quantum confinement in the z-direction (growth direction). The challenge is to provide quantum confinement in the lateral direction. Several fabrication and growth techniques have been investigated for obtaining lateral confinement of the device active region. For example, quantum well structures have been patterned using lithographic and etching techniques, growth has been conducted on patterned substrates or substrates with preferred crystallographic planes. Currently, the most successful approach to achieve defect-free, multiple-layer, high-density, QD ensembles is the heteroepitaxy of coherently-strained, selfassembled islands [29-35], a process referred to as the Stranski-Krastanow (S-K) growth mode [36, 37].

The S-K growth mode is a form of strained-layer epitaxy and is limited by both strain energy and dislocation formation energy. In other words, for a lattice mismatch in the range from $1.8-10 \%$, it is possible to grow a pseudomorphic, two-dimensional epi-layer, or wetting layer, that is lattice matched to the substrate for very small thicknesses. Beyond some critical thickness, the strain energy in the system is relaxed by the formation of misfit dislocations at the epi-layer/substrate interface. However, as long as the epi-layer is below the critical thickness, the strain is absorbed coherently and minimal dislocations are produced by the formation of coherently-strained, three-dimensional islands on the epi-layer surface [38].

For growth along [100] direction in the InAs/GaAs QD system, that lattice mismatch is $7 \%$ and the self-assembled QDs can be lens- or pyramidal-shaped [39]. The typical InAs growth rate is 0.1 monolayer (ML)/sec, and the GaAs substrate temperature is usually cooled to $500{ }^{\circ} \mathrm{C}$. The wetting layer thickness in the InAs/GaAs QD system is $1.7 \mathrm{ML}$, where $1 \mathrm{ML}$ $\sim 2.83 \AA$ for III-V materials. The transition from the two-dimensional wetting layer to threedimensional islands is observed using in-situ reflection high-energy electron diffraction (RHEED), in which a streaky pattern gives way to a spotty pattern when QDs begin to form. Once this transition is observed, InAs overgrowth occurs for 2-5 seconds to provide enough InAs charge (2.2 ML) for pyramidal QDs, and a 30 second growth-interrupt pause is used to allow the complete formation of QDs. After this pause, an intrinsic GaAs cap layer $(\sim 25-60$ $\mathrm{nm}$ ) is grown on top of the InAs QDs, thereby completing the QD potential barrier. This sequence of growth is repeated to achieve a specified number of QD layers. Typically, the QDs are directly-doped with silicon in order to provide free electrons for photoexcitation, and the carrier concentration ranges from $0.5-1 \times 10^{18} \mathrm{~cm}^{-3}$. The doping density provides two electrons per dot for typical InAs/GaAs QD surface densities. For typical growth parameters, an ensemble of InAs/GaAs QDs has lateral sizes from 15-25 nm and heights from 5-8 nm. The surface density of InAs/GaAs QDs ranges from $10^{9}-10^{11} \mathrm{~cm}^{-2}$. An atomic force microscopy image of an ensemble of InAs/GaAs QDs grown by $\mathrm{MBE}$ at $500^{\circ} \mathrm{C}$ at a rate of $0.1 \mathrm{ML} / \mathrm{s}$ is shown in Fig. 3, where the QD surface density is estimated to be $1 \times 10^{11} \mathrm{~cm}^{-2}$. 


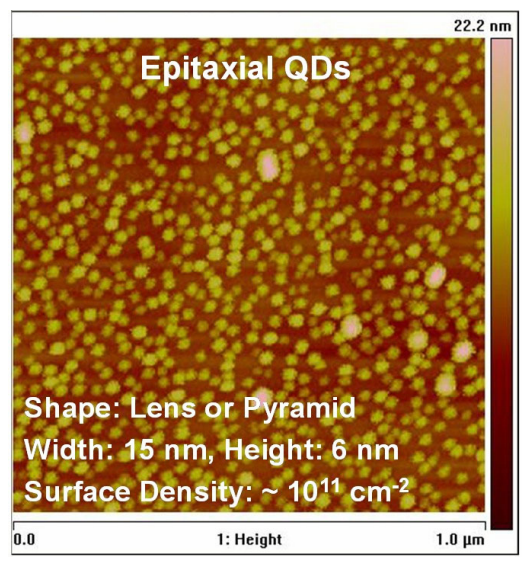

Fig. 3. Planar atomic force microscopy image of epitaxial InAs/GaAs QDs demonstrating important structural characteristics.

\subsection{Intraband absorption in QDs}

The first observation of intraband absorption in InAs/GaAs QDs [40] reported absorption in the 13-15 $\mu \mathrm{m}$ spectral range. In general, there are two types of intraband transitions that can originate in the QD: (i) bound-to-bound transitions in which ground state electrons are photoexcited to the excited state of the QD and (ii) bound-to-continuum transitions in which an electron in the ground- or excited-state is photoexcited out of the QD into the continuum of energy levels near the barrier bulk material band-edge.

The absorption coefficient is an important quantity for determining the applicability of a given QD active region to the detection of IR light. It is clear that as the dimensionality of the active region is reduced, the energy levels and density of states become quantized and the electron distribution is maximized near the conduction band-edge. However, the QD absorption spectrum is generally broad due to the size variation of QDs. In fact, QDIPs experience a tradeoff between absorption strength and absorption linewidth. This trade-off occurs because the size of QDs varies spatially such that the density of states is actually represented by inhomogeneously broadened $\delta$-functions in a Gaussian distribution. For example, a perfectly uniform QD ensemble will have large peak absorption and a narrow absorption linewidth since all the dots experience peak absorption at the same wavelength. However, a non-uniform QD ensemble will have reduced peak absorption and a broad absorption linewidth since many dots with varying sizes experience peak absorption at different wavelengths. Thus, the peak absorption coefficient, $\alpha$ (measured in $\mathrm{cm}^{-1}$ ), is inversely proportional to the density of states inhomogeneous Gaussian linewidth, $\sigma$ (measured in $\mathrm{meV}$ ) [41].

Another important aspect of intraband absorption in QDs is related to the polarization selection rules for incident radiation. In conjunction with the polarization of the electric field, the momentum matrix element determines the selection rules for permitted transitions in which photons are absorbed. An optical transition is forbidden if the dot product between the electric field polarization and the momentum matrix element is zero, since the resulting transition rate would equal zero. The momentum matrix element for intraband transitions is calculated using the electron wavefunctions determined by the 8-band $\mathbf{k} \cdot \mathbf{p}$ model in pyramidal QDs. The 8-band k.p theory is used to determine the electronic bandstructure in semiconductor heterostructures near the band-edge only. An important advantage of this approach is the consideration of remote bands (nearest eight bands with sufficiently small energy differences from the given state [42]), which is particularly important for strained systems such as QD heterostructures. In fact, it has been demonstrated experimentally that 
the large built-in strain of InAs/GaAs QDs causes a significant change in the properties of the electronic bandstructure. For example, the bandgap of InAs/GaAs QDs has been measured to be $\sim 1.05 \mathrm{eV}$, even though the intrinsic bandgap of bulk InAs is $0.4 \mathrm{eV}$ [39]. In QD devices, the polarization selection rules are relaxed compared to quantum wells, and lateral- or normal-incidence IR light may be used for intraband transitions to occur. This flexibility results from the fact that QDs experience three-dimensional quantum confinement, and therefore conserve momentum for intraband transitions in any direction.

\subsection{Challenges of InAs/GaAs QDIPs: dopant incorporation}

While the three-dimensional quantum confinement of the active region in QDIPs is expected to provide many advantages, in practice, it has been a challenge to meet all of these expectations. This discrepancy between predicted and realized advantages is due in large part to the epitaxial growth mechanism of QDs. In fact, one of the most significant consequences of the S-K growth mode is the random fluctuation of dot size, material composition, and doping. This non-uniformity of QDs leads to an inherently large full-width half-maximum (FWHM) linewidth $(\sim 50 \mathrm{meV})$ for transitions in a QD ensemble. This large, inhomogeneously broadened linewidth has a deleterious effect on QDIP performance; especially the dark current and spectral response (bound energy level spacing). The S-K growth mode also introduces challenges due to the build-up of strain for repeated dot layers. By improving QD uniformity, the linewidth of the QD ensemble should decrease, thus increasing the absorption coefficient and detector efficiency and improving the performance of QDIPs. Thus the uniform epitaxial growth of QD heterostructures is one of the most important issues related to obtaining state-of-the-art performance in QDIPs and to establishing QDIPs as a mature technology.

QDIP performance could be improved (higher operating temperature and multi-spectral response) by exerting better control over dopant incorporation in QDs. For higher dopant densities, the dark current increases since electrons occupy excited-state energy levels in QDs, enabling these carriers to contribute to the noise in the device more easily by thermionic emission or field-assisted tunneling. For lower dopant densities, the absorption efficiency decreases since there are fewer electrons in the QD ground state available for photoexcitation. For these reasons, it is customary to design QDIPs with doping such that there are approximately two electrons per QD ground state [43]. However, it is not clear how to actually achieve this doping level during growth.

Different doping techniques, doping positions, and doping concentrations have been investigated to optimize QDIP performance [4, 13, 44-52]. An important issue to be addressed is that QDIPs demonstrate higher dark currents, lower activation energies (minimum energy required for an electron to escape from QD), and lower operating temperatures than that predicted theoretically. It has been suggested that this discrepancy is due to defect-assisted, sequential resonant tunneling in Si-doped, InAs/GaAs QDIPs [51, 53]. These dopant-induced defects, known as donor-complex-defect (DX) centers, are thought to exist deep within the bandgap [54-56]. However, Si-induced DX centers in GaAs have multiple, metastable states above the GaAs conduction band edge [57], thereby reducing the feasibility of sequential resonant tunneling from InAs QD states deep within the GaAs bandgap. Nonetheless, the DX center metastable states in GaAs can be occupied by electrons that are energized by an external field $[58,59]$. Therefore, the observed reduction in QDIP activation energy could be related to a dipole field between positively charged DX centers and electrons in QDs that induces tunneling from confined energy levels in the QD. Therefore, electrons can escape with much smaller activation energies in doped QDIPs.

DX centers in GaAs due to Si-doping have been shown to exist by photocapacitance-time spectra, which demonstrate quenching in the presence of DX center metastable states [60]. Variable temperature $(35-300 \mathrm{~K})$ dark current-voltage measurements have been used to 
determine the QD (doped and undoped) activation energy as a function of bias voltage by the use of Arrhenius plots [60]. For doped QDs, there is a dramatic decrease in the activation energy with increasing bias compared to undoped QDs. In fact, three bias-dependent regions can be defined for the activation energy of the doped QDs. In Region I, the QD activation energy is dominated by thermal activation from the InAs QD to the GaAs conduction band edge. In Region II, the activation energy of the doped samples experiences a dramatic decrease due to a dipole field formed between DX centers and electrons in the QDs. In Region III, the dipole field becomes the dominant factor in electron transport. This DX center, dipole-induced tunneling mechanism has a profound effect on dark current, and its identification should lead to better understanding of QDIP device performance [60-62].

\section{MID-INFRARED QDIPS: A NICHE MARKET}

Despite the challenges associated with QDIPs, these detectors possess two important characteristics that motivate their investigation and distinguish them from state-of-the-art thermal detectors (such as microbolometers) and low-bandgap photodiodes (such as $\mathrm{HgCdTe}$ ). First, while thermal detectors operate at room temperature and are inexpensive, lightweight, and commercially available; IR photodetection is still preferred for advanced applications requiring high spectral resolution and fast modulation response, such as military IR signature analysis. Second, low bandgap semiconductors, such as $\mathrm{HgCdTe}$, are inherently soft and brittle. Thus, it can be difficult to epitaxially grow and fabricate IR photodetectors with low-bandgap, bulk material active regions, and the yield of these devices tends to be low. Compared to HgCdTe bulk photodiodes, QDIPs are particularly well-suited for detecting multiple wavelengths of light simultaneously in a single device heterostructure, thereby increasing the spectral resolution; and QDIPs have a faster response to changing input since the carrier lifetime is not limited by diffusion (which is typically a slow mechanism). Thus, the demonstration of a multi-spectral QDIP at high operating temperature with fast modulation response and device characteristics comparable to HgCdTe bulk photodiodes constitutes a performance threshold to justify commercialization. According to these criteria, research conducted to date demonstrates that QDIPs have the potential to fulfill a niche market in high-temperature $(\geq 150 \mathrm{~K})$, mid-IR (3-5 $\mu \mathrm{m})$ photodetection.

\subsection{State-of-the-art performance in InAs/GaAs QDIPs}

State-of-the-art performance in InAs/GaAs QDIPs has been demonstrated for single devices and arrays [11, 16-28]. Important figures of merit for single devices include dark current density, peak responsivity, and peak detectivity. These device characteristics must meet given specifications to ensure compatibility with a silicon read-out integrated circuit (ROIC) in the fabrication of focal plane arrays (FPAs). Furthermore, the QDIP operating temperature is important to consider because it motivates the investigation of QD active regions. Therefore, as an example of state-of-the-art performance in the mid-IR, the figures of merit for a 70layer InAs/GaAs QDIP are presented [24, 46]. The large number of QD layers in this device served to increase IR absorption, and GaAs barriers $(\geq 50 \mathrm{~nm})$ were required to reduce dislocation propagation in the large QD stack. Table 1 shows the measured QDIP performance at $150 \mathrm{~K}$ (for peak spectral response at $4.7 \mu \mathrm{m}$ ) and the required QDIP performance for mid-IR FPA fabrication at JPL using a (350x256) Si ROIC manufactured by Indigo Systems. It is important to note that the dark current and peak responsivity requirements were met. In the case of the peak detectivity, the largest QDIP value obtained $\left(\sim 10^{10} \mathrm{~cm} \mathrm{~Hz}^{1 / 2} / \mathrm{W}\right)$ was two orders of magnitude below the desired value. However, this performance at high operating temperature, which is comparable to $\mathrm{HgCdTe}$ photodiodes, helped establish InAs/GaAs QDIPs as a viable technology for IR FPAs. 
Table 1. Comparison of 70-layer QDIP performance to FPA requirements.

\begin{tabular}{|c|c|c|c|}
\hline & $\begin{array}{c}\mathrm{J}_{\text {dark }} \\
\left(\mathrm{A} / \mathrm{cm}^{2}\right)\end{array}$ & $\begin{array}{l}\mathrm{R}_{\text {peak }} \\
(\mathrm{A} / \mathrm{W})\end{array}$ & $\begin{array}{c}\mathrm{D}^{*}{ }_{\text {peak }} \\
\left(\mathrm{cmHz}^{1 / 2} / \mathrm{W}\right)\end{array}$ \\
\hline $\begin{array}{c}\text { 70-layer QDIP } \\
\text { (Peak Spectral } \\
\text { Response }-4.7 \mu \mathrm{m} \text {; } \\
\text { Temp }-150 \mathrm{~K} \\
\text { Bias }-2.0 \mathrm{~V} \text { ) }\end{array}$ & $5.80 \times 10^{-5}$ & 0.201 & $3.7 \times 10^{10}$ \\
\hline $\begin{array}{c}\text { QDIP FPA } \\
\text { Requirements }\end{array}$ & $\sim 10^{-5}$ & $\sim 0.100$ & $\sim 10^{12}$ \\
\hline
\end{tabular}

State-of-the-art array performance has been demonstrated by IR imaging using QDIP FPAs [26-28]. Some of these FPAs feature QD material systems other than InAs/GaAs, and these active regions are discussed in the following section. Table 2 shows important QDIP FPA figures of merit for three different array demonstrations. Table 3 shows comparable performance parameters for commercially-available IR detector technologies (microbolometer [63], HgCdTe [64], quantum well IR photodetector [65]). It is important to note that QDIP FPAs are approaching the same performance as other available technologies.

Table 2. QDIP FPA performance.

\begin{tabular}{|c|c|c|c|}
\hline & $\begin{array}{c}\text { Northwestern } \\
\text { University }(2004)(26)\end{array}$ & $\begin{array}{l}\text { Chung-Shan Institute of } \\
\text { Science and } \\
\text { Technology, Taiwan } \\
(2006)(27)\end{array}$ & $\begin{array}{c}\text { Jet Propulsion } \\
\text { Laboratory/ U. New } \\
\text { Mexico }(2007)(28)\end{array}$ \\
\hline $\begin{array}{c}\text { Sensor } \\
\text { Material }\end{array}$ & InGaAs/InGaP QDIP & InAs/GaAs QDIP & $\begin{array}{c}\text { InAs/InGaAs/GaAs } \\
\text { DWELL QDIP }\end{array}$ \\
\hline Spectral Peak & $4.7 \mu \mathrm{m}$ & $5.2 \mu \mathrm{m}$ & $8.1 \mu \mathrm{m}$ \\
\hline Array Size & $256 \times 256$ & $256 \times 256$ & $640 \times 512$ \\
\hline $\begin{array}{l}\text { Operating } \\
\text { Temperature }\end{array}$ & $77 \mathrm{~K}$ & $80 \mathrm{~K}$ & $77 \mathrm{~K}$ \\
\hline $\begin{array}{c}\text { Peak } \\
\text { Detectivity }\end{array}$ & $3.7 \times 10^{10} \mathrm{cmHz}^{1 / 2} / \mathrm{W}$ & $1.5 \times 10^{10} \mathrm{cmHz}^{1 / 2} / \mathrm{W}$ & $1 \times 10^{10} \mathrm{cmHz}^{1 / 2} / \mathrm{W}$ \\
\hline
\end{tabular}

Table 3. Commercially-available IR detector technologies.

\begin{tabular}{cccc}
\hline & $\begin{array}{c}\text { Core by Indigo } \\
\text { Photon 320(63) }\end{array}$ & $\begin{array}{c}\text { FLIR/Cedip } \\
\text { Titanium Orion } \\
\text { Model } 890(64)\end{array}$ & $\begin{array}{c}\text { FLIR } \\
\text { SC4000(65) }\end{array}$ \\
\hline Sensor Material & Vanadium Oxide & HgCdTe Photodiode & $\begin{array}{c}\text { GaAs Quantum Well IR } \\
\text { Photodetector }\end{array}$ \\
Spectral Range & $7.5-13.5 \mu \mathrm{m}$ & $7.7-11 \mu \mathrm{m}$ & $8.0-9.2 \mu \mathrm{m}$ \\
Array Size & $324 \times 256$ & $320 \times 256$ & $320 \times 256$ \\
Cooling System & Uncooled & Stirling Cooler & Stirling Cooler \\
Sensitivity & $\leq 85 \mathrm{mK}$ & $<20 \mathrm{mK}$ & $<35 \mathrm{mK}$ \\
(NEDT) & $\geq 98 \%$ & $>99.5 \%$. & $>99.5 \%$ \\
Operability & $30 \mathrm{~Hz}$ & 1 to $380 \mathrm{~Hz}$ & 1 to $420 \mathrm{~Hz}$ \\
Frame Rate & & &
\end{tabular}




\section{MOVING BEYOND THE STANDARD INAS/GAAS QDIP}

\subsection{Alternative QD material systems}

While the InAs/GaAs QD is a fundamental material system upon which much progress has been made in QDIP performance, there are many other material options for use in these devices. In fact, an important advantage of the QDIP is that the device spectral response can be tuned throughout the IR spectrum (from $\sim 50$ to $500 \mathrm{meV}$ ) by varying the QD heterostructure [66-72]. One variation of InAs/GaAs QDs involves including Ga in the InAs $\mathrm{QD}$ and/or $\mathrm{Al}$ in the GaAs barrier [6, 46,73,74]. AlGaAs is advantageous as a barrier material due to its larger bandgap that helps reduce dark current. Also, $\mathrm{In}_{0.4} \mathrm{Ga}_{0.6} \mathrm{As} / \mathrm{GaAs}$ QDs have been used in three-color QDIPs, demonstrating IR responses at 3.5, 7.5, and $22 \mu \mathrm{m}$ for different bias voltages [21]. Other III-V material systems under investigation include InAs/InP QDs [75] and InAs/InAlAs QDs [76] grown on InP substrates and InGaAs/InGaP QDs grown on GaAs substrates [77]. Recent results have demonstrated a mid-IR QDIP (4.7 $\mu \mathrm{m}$ ) using $\mathrm{In}_{0.68} \mathrm{Ga}_{0.32} \mathrm{As} / \mathrm{In}_{0.49} \mathrm{Ga}_{0.51} \mathrm{P}$ QDs grown on GaAs substrates by MOCVD [78]. The device performance demonstrated peak responsivity of $1.2 \mathrm{~A} / \mathrm{W}$ amd specific peak detectivity of $1.1 \times 10^{12} \mathrm{cmHz}^{1 / 2} \mathrm{~W}^{-1}$ at $77 \mathrm{~K}$. This high performance is credited to the reduction of relaxed QDs in the active region and the optimization of doping. Intraband transitions in the IR have also been observed for Ge/Si QDs [79-81]. These boron-doped devices use holes trapped in the QD valence band for the photogeneration of carriers, and the characterization of $\mathrm{Ge} / \mathrm{Si}$ QDIPs indicates they may be an important technology for far IR detection (> $14 \mu \mathrm{m})$ [82-85].

\subsection{QDIP bandstructure engineering}

In addition to changing the QD material system, another option is to engineer the QDIP bandstructure to strategically enable different device capabilities. For example, a common feature in state-of-the-art InAs/GaAs QDIPs is the AlGaAs current-blocking barrier layer directly beneath the top ohmic contact. In this way, significant reductions in dark current have been achieved because the AlGaAs barrier blocks thermionic emission [11, 12], and the operating temperature of QDIPs has increased. Two additional examples of bandstructure engineering include dot-in-a-well (DWELL) and resonant-tunneling (RT) heterostructures.

InAs QDs embedded in strain-relieving InGaAs quantum wells are known as DWELL heterostructures [86]. The interaction between these quantum-confined structures and the modified strain effects within the quantum dot serve to provide alternate intraband energy transitions. InAs/ $/ \mathrm{In}_{0.15} \mathrm{Ga}_{0.85}$ As DWELL systems have been successful in detecting LWIR light [16]. QDIPs using DWELLs not only permit greater control over detection wavelength tunability [87], but they have also demonstrated excellent device performance [88].

RT-QDIPs use resonant tunneling filters to selectively block dark current while transmitting photocurrent $[19,89-92]$. When the thickness and position of a double AlGaAs barrier are properly designed around a QD layer, the electron tunneling probability is approximately 1 at a particular energy corresponding to the desired peak detection wavelength. At the same time, the tunneling probability is several orders of magnitude lower for energies that are several $\mathrm{meV}$ removed from the peak energy. Thus, since dark current is due to electrons with a broad energy distribution, the dark current will be significantly reduced by the resonant tunneling filter. The RT-QDIP has demonstrated a significant improvement in terms achieving high operating temperatures, demonstrating device operation at temperatures as high as $300 \mathrm{~K}$.

RT-QDIPs are especially promising as far-IR and terahertz photodetectors due to the suppression of dark current at higher operating temperatures [93, 94]. A RT-QDIP using an In0.6Alo.4As/GaAs QD active region has demonstrated spectral response peaking at $50 \mu \mathrm{m}$, with peak responsivity of $0.45 \mathrm{~A} / \mathrm{W}$ and specific detectivity of $10^{8} \mathrm{~cm} \mathrm{~Hz}^{1 / 2} / \mathrm{W}$ (bias $=1 \mathrm{~V}$ ) 
[94]. While this device performance was obtained at $4.6 \mathrm{~K}, \mathrm{THz}$ spectral response was observed at temperatures as high as $150 \mathrm{~K}$. It is also interesting to note that RT-DWELLQDIPs have been demonstrated, yielding a reduction in dark current by two orders of magnitude and an increase in peak detectivity by five times compared to a DWELL control device [95].

\subsection{Colloidal QDIPs}

A fundamentally different approach to QDIPs is to use an active region with threedimensional quantum confined semiconductor nanoparticles synthesized by inorganic chemistry [96]. As an example, Fig. 4 shows a planar transmission electron microscopy image of $\mathrm{CdSe} / \mathrm{CdS}$ nanoparticles, demonstrating the typical size and shape. These nanoparticles, also known as colloidal QDs, could improve QDIP performance compared to epitaxial QDs due to: (i) control over colloidal QD synthesis and ability to conduct size-filtering, leading to highly-uniform ensembles, (ii) spherical shape of colloidal QDs, simplifying calculations for device modeling and design, and (iii) greater selection of active region materials since strain considerations that dominate the growth of epitaxial QDs are eliminated. Typically, colloidal QDs are applied to optoelectronic devices as conducting-polymer/nanocrystal blends, or nanocomposites [97, 98]. Colloidal QD solids have also been investigated [99, 100]. For IR photodetector applications, nanocomposites often feature narrow-bandgap, II-VI, PbSe or $\mathrm{PbS}$ colloidal QDs. IR photodetectors that have been reported using colloidal QDs embedded in conducting polymer matrices, such as poly[2-methoxy-5-(2-ethylhexyloxy)-1,4phenylenevinylene] (MEH-PPV), demonstrate photodetection in the near-IR regime $(1-3 \mu \mathrm{m})$ corresponding to the semiconductor nanocrystal bandgap energy [101-104]. Colloidal QD photodetectors typically comprise a single nanocomposite layer deposited on a glass slide by spin-casting, and large-area, two-terminal, vertical devices are fabricated using p- (indiumtin-oxide) and n-type (aluminum) contacts, as shown schematically in Fig. 5 for a PbS/MEHPPV nanocomposite.

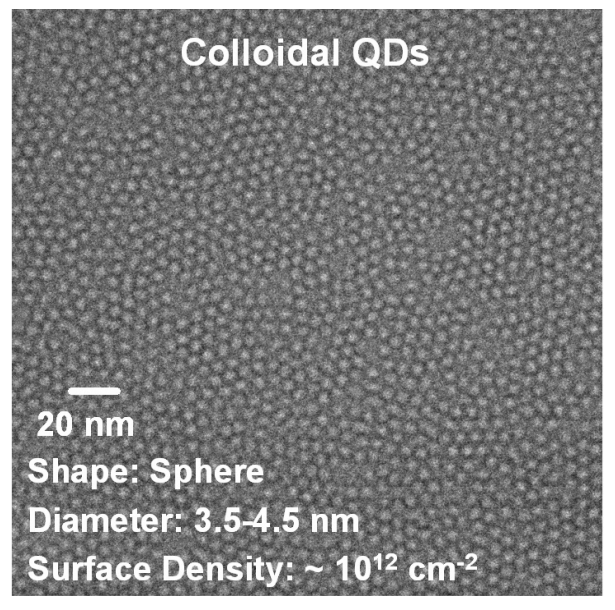

Fig. 4. Planar transmission electron microscopy image of $\mathrm{CdSe} / \mathrm{CdS}$ core-shell colloidal QDs demonstrating important structural characteristics, such as increased uniformity across the ensemble. 


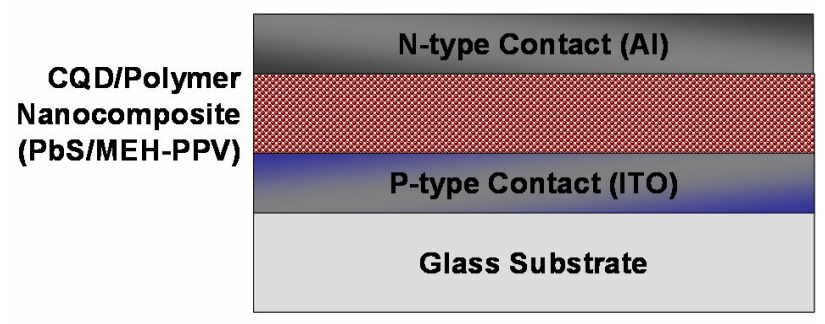

Fig. 5. Schematic diagram of device heterostructure in colloidal PbS QD/ MEH$\mathrm{PPV}$ conducting polymer nanocomposites for infrared photodetection.

It is important to note that colloidal QD nanocomposites exhibit subtle differences compared to epitaxial QDIPs. First, intraband transitions are not exploited. Instead, bipolar, interband (or excitonic) transitions across the colloidal QD bandgap contribute to the photoresponse of the detector. In addition, since colloidal QDs are electron acceptors and the polymers are typically hole conductors, the photogenerated excitons are dissociated at the $\mathrm{QD} /$ polymer interface. Thus, photoconduction through the nanocomposite occurs as electrons hop among QDs and holes transport through the polymer. This IR photodetection mechanism is shown schematically in Fig. 6.

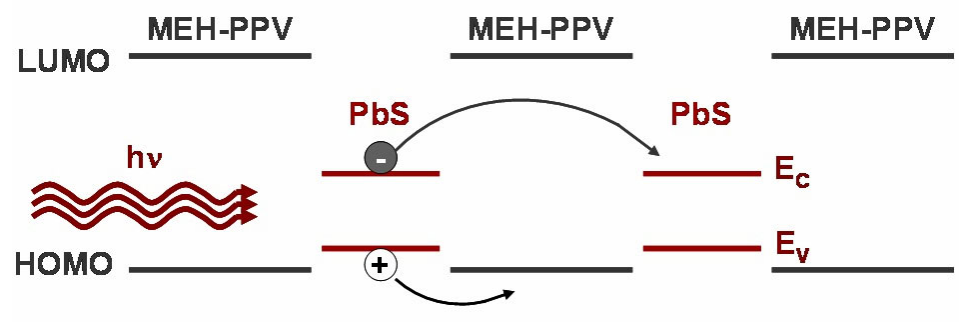

Fig. 6. Schematic diagram of energy vs. position for interband transitions in $\mathrm{PbS} / \mathrm{MEH}-\mathrm{PPV}$ colloidal QD-conducting polymer nanocomposites demonstrating photocurrent generation for IR photodetection.

\section{CONCLUSIONS}

QDIPs are positioned to become an important technology in the field of IR detection, particularly for the type of high-temperature, low-cost, high-yield detector arrays required for the military and other highly sophisticated applications. State-of-the-art mid-IR detection at $150 \mathrm{~K}$ has been demonstrated using 70-layer InAs/GaAs QDIPs, and QDIP FPAs are approaching performance comparable to $\mathrm{HgCdTe}$ at $77 \mathrm{~K}$. In particular, QDIPs have the potential to fulfill a niche market in high-temperature mid-IR photodetection, and it is reasonable to consider incorporating these devices into IR camera systems. By addressing critical challenges inherent to epitaxial QD material systems, such as controlling dopant incorporation, and by exploring alternative QD systems, such as colloidal QDs, the performance and applicability of QDIPs can be enhanced even more.

\section{Acknowledgments}

The author acknowledges support from the National Science Foundation, the Air Force Office of Scientific Research, and the Office of Naval Research. 


\section{References}

[1] J. Phillips, P. Bhattacharya, S. W. Kennerly, D. W. Beekman, and M. Dutta, "Selfassembled InAs-GaAs quantum-dot intersubband detectors," IEEE J. Quant. Electron. 35, 936-943 (1999) [doi:10.1109/3.766837].

[2] V. Ryzhii, "The theory of quantum-dot infrared phototransistors," Semicond. Sci. Technol. 11, 759-765 (1996) [doi:10.1088/0268-1242/11/5/018].

[3] K. W. Berryman, S. A. Lyon, and M. Segev, "Mid-infrared photoconductivity in InAs quantum dots," Appl. Phys. Lett. 70, 1861-1863 (1997) [doi:10.1063/1.118714].

[4] J. Phillips, K. Kamath, and P. Bhattacharya, "Far-infrared photoconductivity in selforganized InAs quantum dots," Appl. Phys. Lett. 72, 2020-2022 (1998) [doi:10.1063/1.121252].

[5] D. Pan, E. Towe, and S. Kennerly, "Normal-incidence intersubband (In, Ga)As/GaAs quantum dot infrared photodetectors," Appl. Phys. Lett. 73, 1937-1939 (1998) [doi:10.1063/1.122328].

[6] S. J. Xu, S. J. Chua, T. Mei, X. C. Wang, X. H. Zhang, G. Karunasiri, W. J. Fan, C. H. Wang, J. Jiang, S. Wang, and X. G. Xie, "Characteristics of InGaAs quantum dot infrared photodetectors," Appl. Phys. Lett. 73, 3153-3155 (1998) [doi:10.1063/1.122703].

[7] S. Maimon, E. Finkman, G. Bahir, S. E. Schacham, J. M. Garcia, and P. M. Petroff, "Intersublevel transitions in InAs/GaAs quantum dots infrared photodetectors," Appl. Phys. Lett. 73, 2003-2005(1998) [doi:10.1063/1.122349].

[8] D. Pan, E. Towe, and S. Kennerly, "Photovoltaic quantum-dot infrared detectors," Appl. Phys. Lett. 76, 3301-3303(2000) [doi:10.1063/1.126613].

[9] A. D. Stiff, S. Krishna, P. Bhattacharya, and S. Kennerly, "Normal-incidence, hightemperature, mid-infrared InAs-GaAs vertical quantum-dot infrared photodetector," IEEE J. Quant. Electron. 37, 1412-1419 (2001) [doi:10.1109/3.958360].

[10] H. C. Liu, M. Gao, J. McCafferey, Z. R. Wasilewski, and S. Fafard, "Quantum dot infrared photodetectors," Appl. Phys. Lett. 78, 79-81 (2001) [doi:10.1063/1.1337649].

[11] S. Y. Wang, S. D. Lin, H. W. Wu, and C. P. Lee, "Low dark current quantum-dot infrared photodetectors with an AlGaAs current blocking layer," Appl. Phys. Lett. 78, 1023-1025 (2001) [doi:10.1063/1.1347006].

[12] S.-F. Tang, S.-Y. Lin, and S.-C. Lee, "Near-room-temperature operation of an InAs/GaAs quantum-dot infrared photodetector," Appl. Phys. Lett. 78, 2428-2430 (2001) [doi:10.1063/1.1362201].

[13] Z. Chen, O. Baklenov, E. T. Kim, I. Mukhametzhanov, J. Tie, A. Madhukar, Z. Ye, and J. C. Campbell, "Normal incidence InAs/AlxGa1-xAs quantum dot infrared photodetectors with undoped active region," J. Appl. Phys. 89, 4558-4563 (2001) [doi:10.1063/1.1356430].

[14] S. Krishna, A. D. Stiff-Roberts, J. D. Phillips, P. Bhattacharya, and S. W. Kennerly, "Hot dot detectors: infrared quantum dot intersubband photodetectors are a promising technology for multiwavelength IR detection," IEEE Circ. Dev. Mag. 18, 14-24 (2002) [doi:10.1109/MCD.2002.981296].

[15] Y. H. Kang, J. Park, U. H. Lee, and S. Hong, "Effect of the dot size distribution on quantum dot infrared photoresponse and temperature-dependent dark current," Appl. Phys. Lett. 82, 1099-1101 (2003) [doi:10.1063/1.1555711].

[16] S. Krishna, S. Raghavan, G. v. Winckel, P. Rotella, A. Stintz, C. P. Morath, D. Le, and S. W. Kennerly, "Two color InAs/InGaAs dots-in-a-well detector with background-limited performance at 91 K," Appl. Phys. Lett. 82, 2574-2576 (2003) [doi:10.1063/1.1567806]. 
[17] S. Chakrabarti, A. D. Stiff-Roberts, X. H. Su, P. Bhattacharya, G. Ariyawansa, and A. G. U. Perera, "High-performance mid-infrared quantum dot infrared photodetectors," J. Phys. D: Appl.Phys. 38, 2135-2141 (2005) [doi:10.1088/00223727/38/13/009].

[18] A. D. Stiff-Roberts, X. H. Su, S. Chakrabarti, and P. Bhattacharya, "Contribution of field-assisted tunneling emission to dark current in InAs/GaAs quantum dot infrared photodetectors," IEEE Photon. Technol. Lett. 16(3), 867-869 (2004) [doi:10.1109/LPT.2004.823690].

[19] X. H. Su, S. Chakrabarti, P. Bhattacharya, G. Ariyawansa, and A. G. U. Perera, "A resonant tunneling quantum dot infrared photodetector," IEEE J. Quant. Electron. 41(7), 974-979 (2005) [doi:10.1109/JQE.2005.848901].

[20] U. Sakoglu, J. S. Tyo, M. M. Hayat, S. Raghavan, and S. Krishna, "Spectrally adaptive infrared photodetectors with bias-tunable quantum dots," J. Opt. Soc. Am. B 21(1), 7-17 (2004) [doi:10.1364/JOSAB.21.000007].

[21] S. Chakrabarti, X. H. Su, P. Bhattacharya, G. Ariyawansa, and A. G. U. Perera, "Characteristics of a multi-color InGaAs/GaAs quantum dot infrared photodetector," IEEE Photon. Technol. Lett. 17, 178-180 (2005) [doi:10.1109/LPT.2004.838295].

[22] J. Jiang, S. Tsao, T. O'Sullivan, W. Zhang, H. Lim, T. Sills, K. Mi, M. Razeghi, G. J. Brown, and M. Z. Tidrow, "High detectivity InGaAs/InGaP quantum-dot infrared photodetectors grown by low pressure metalorganic chemical vapor deposition," Appl. Phys. Lett. 84(12), 2166-2168 (2004) [doi:10.1063/1.1688982].

[23] J.-W. Kim, J.-E. Oh, S.-C. Hong, C.-H. Park, and T.-K. Yoo, "Room temperature far infrared (8-10 um) photodetectors using self-assembled InAs quantum dots with high detectivity," IEEE Electron Dev. Lett. 21(7), 329-331 (2000) [doi:10.1109/55.847370].

[24] S. Chakrabarti, A. D. Stiff-Roberts, P. Bhattacharya, S. Gunapala, S. Bandara, S. B. Rafol, and S. W. Kennerly, "High-temperature operation of InAs-GaAs quantum-dot infrared photodetectors with large responsivity and detectivity," IEEE Photon. Technol. Lett. 16(5), 1361-1363 (2004) [doi:10.1109/LPT.2004.825974].

[25] A. D. Stiff-Roberts, S. Chakrabarti, S. Pradhan, B. Kochman, and P. Bhattacharya, "Raster-scan imaging with normal-incidence, mid-infrared InAs/GaAs quantum dot infrared photodetectors," Appl. Phys. Lett. 80, 3265-3267 (2002) [doi:10.1063/1.1476387].

[26] J. Jiang, K. Mi, S. Tsao, W. Zhang, H. Lim, T. O'Sullivan, T. Sills, M. Razeghi, G. J. Brown, and M. Z. Tidrow, "Demonstration of a 256x256 middle-wavelength infrared focal plane array based on InGaAs/InGaP quantum dot infrared photodetectors," Appl. Phys. Lett. 84, 2232-2234 (2004) [doi:10.1063/1.1688000].

[27] S.-F. Tang, C.-D. Chiang, P.-K. Weng, Y.-T. Gau, J.-J. Luo, S.-T. Yang, C.-C. Shih, S.-Y. Lin, and S.-C. Lee, "High-temperature operation normal incident 256256 InAs-GaAs quantum-dot infrared photodetector focal plane array," IEEE Photon. Technol. Lett. 18, 986-988 (2006) [doi:10.1109/LPT.2006.873458].

[28] S. D. Gunapala, S. V. Bandara, C. J. Hill, D. Z. Ting, J. K. Liu, S. B. Rafol, E. R. Blazejewski, J. M. Mumolo, S. A. Keo, S. Krishna, Y.-C. Chang, and C. A. Shott, "640 512 pixels long-wavelength infrared (LWIR) quantum-dot infrared photodetector (QDIP) imaging focal plane array," IEEE J. Quant. Electron. 43, 230237 (2007) [doi:10.1109/JQE.2006.889645].

[29] L. Goldstein, F. Glas, J. Y. Marzin, M. N. Charasse, and G. LeRoux, "Growth by molecular beam epitaxy and characterization of $\mathrm{InAs} / \mathrm{GaAs}$ strained-layer superlattices," Appl. Phys. Lett. 47, 1099-1101 (1985) [doi:10.1063/1.96342].

[30] P. R. Berger, K. Chang, P. K. Bhattacharya, and J. Singh, "A study of strain-related effects in the molecular-beam epitaxy growth of $\mathrm{In}_{\mathrm{x}} \mathrm{Ga}_{1-\mathrm{x}} \mathrm{As}$ on GaAs using reflection 
high-energy electron diffraction," J. Vac. Sci. Technol. B 5, 1162-1166 (1987) [doi:10.1116/1.583704].

[31] M. Tabuchi, S. Noda, and A. Sasaki, "Strain energy and critical thickness of heteroepitaxial InGaAs layers on GaAs substrate," J. Cryst. Growth 115, 169 (1991) [doi:10.1016/0022-0248(91)90733-L].

[32] D. Leonard, M. Krishnamurthy, C. M. Reaves, S. P. Denbaars, and P. M. Petroff, "Direct formation of quantum-sized dots from uniform coherent islands of InGaAs on GaAs surfaces," Appl. Phys. Lett. 63, 3203-3205 (1993) [doi:10.1063/1.110199].

[33] Q. Xie, P. Chen, A. Kalburge, T. R. Ramachandran, A. Nayfonov, A. Konkar, and A. Madhukar, "Realization of optically active strained InAs island quantum boxes on GaAs (100) via molecular beam epitaxy and the role of island induced strain fields," J. Cryst. Growth 150, 357-363 (1995) [doi:10.1016/0022-0248(95)80235-5].

[34] D. Bimberg, M. Grundmann, and N. N. Ledentsov, "Growth, spectroscopy, and laser application of self-ordered III-V quantum dots," Mater. Res. Soc. Bull. 23, 31-34 (1998).

[35] P. Bhattacharya, K. Kamath, J. Phillips, and D. Klotzkin, "Self-organized growth of In(Ga)As/GaAs quantum dots and their opto-electronic device applications," Bull. Mater. Sci. 22(3), 519-529 (1999) [doi:10.1007/BF02749964].

[36] I. N. Stranski and L. Krastanow, "Theory of orientation separation of ionic crystals," Akad. Wissen.Wien Math. Natur. Kl. Abt. IIb. 146, 797-810 (1938).

[37] D. Bimberg, M. Grundmann, and N. N. Ledentsov, Quantum Dot Heterostructures, Wiley, Chichester, United Kingdom(1999).

[38] P. R. Berger, K. Chang, P. Bhattacharya, J. Singh, and K. K. Bajaj, "Role of strain and growth conditions on the growth front profile of $\operatorname{In}_{\mathrm{x}} \mathrm{Ga}_{1-\mathrm{x}} \mathrm{As}$ on $\mathrm{GaAs}$ during the pseudomorphic growth regime," Appl. Phys. Lett. 53(8), 684-686 (1988) [doi:10.1063/1.99850].

[39] H. Jiang and J. Singh, "Strain distribution and electronic spectra of InAs/GaAs selfassembled dots: an eight-band study," Phys. Rev. B 56, 4696-4701 (1997) [doi:10.1103/PhysRevB.56.4696].

[40] D. Pan, Y. P. Zeng, M. Y. Kong, J. Wu, Y. Q. Zhu, C. H. Zhang, J. M. Li, and C. Y. Wang, "Normal incident infrared absorption from InGaAs/GaAs quantum dot superlattice," Electron. Lett. 32, 1726-1727 (1996) [doi:10.1049/el:19961135].

[41] J. Singh, Electronic and Optoelectronic Properties of Semiconductor Structures, Cambridge University Press, New York (2003).

[42] J. Singh, Physics of Semiconductors and Their Heterostructures, McGraw-Hill, New York (1993).

[43] J. Phillips, "Evaluation of the fundamental properties of quantum dot infrared detectors," J. Appl. Phys. 91, 4590-4594 (2002) [doi:10.1063/1.1455130].

[44] N. Horiguchi, T. Futatsugi, Y. Nakata, N. Yokoyama, T. Mankad, and P. M. Petroff, "Quantum dot infrared photodetector using modulation doped InAs self-assembled quantum dots," Jpn. J. Appl. Phys. 38, 2559-2561 (1999) [doi:10.1143/JJAP.38.2559].

[45] H. Hwang, K. Park, J.-H. Kang, S. Yoon, and E. Yoon, "Optical properties of Sidoped InAs/InP quantum dots," Curr. Appl. Phys. 3, 465-468 (2003) [doi:10.1016/j.cap.2002.11.002].

[46] A. D. Stiff-Roberts, Design, Fabrication, and Characterization of In $(\mathrm{Ga}, \mathrm{Al}) \mathrm{As} /(\mathrm{Ga}, \mathrm{Al})$ As Quantum Dot Infrared Photodetectors for High-Temperature Operation, PhD Dissertation, Univ. Michigan, Ann Arbor (2004)

[47] E. T. Kim, A. Madhukar, Z. M. Ye, and J. C. Campbell, "High detectivity InAs quantum dot infrared photodetectors," Appl. Phys. Lett. 84, 3277-3279 (2004) [doi:10.1063/1.1719259]. 
[48] U. H. Lee, Y. H. Kang, J. H. Oum, S.-J. Lee, M. Kim, S. K. Noh, Y. D. Jang, D. Lee, H. S. Kim, C. H. Park, and S. Hong, "A study on doping density in InAs/GaAs quantum dot infrared photodetector," Jpn. J. Appl. Phys. 43, 5199-5203 (2004) [doi:10.1143/JJAP.43.5199].

[49] D. Pal and E. Towe, " Uniformly doped InAs/GaAs quantum-dot infrared photodetector structures," J. Vac. Sci. Technol. B 23, 1132-1135 (2005) [doi:10.1116/1.1881572].

[50] R. S. Attaluri, S. Annamalai, K. T. Posani, A. Stintz, and S. Krishna, "Influence of Si doping on the performance of quantum dots-in-a-well photodetectors," J. Vac. Sci. Technol. B 24, 1553-1555 (2006) [doi:10.1116/1.2190676].

[51] K. Drozdowicz-Tomsia, E. M. Goldys, L. Fu, and C. Jagadish, "Doping effect on dark currents in In $0.5 \mathrm{Ga} 0.5 \mathrm{As} / \mathrm{GaAs}$ quantum dot infrared photodetectors grown by metal-organic chemical vapor deposition," Appl. Phys. Lett. 89, 113510 (2006) [doi:10.1063/1.2354432].

[52] Z. Y. Zhao, W. M. Zhang, C. Yi, A. D. Stiff-Roberts, B. J. Rodriguez, and A. P. Baddorf, "Doping characterization of InAs/GaAs quantum dot heterostructure by cross-sectional scanning capacitance microscopy," Appl. Phys. Lett. 92, 092101 (2008) [doi:10.1063/1.2889938].

[53] A. D. Stiff-Roberts, X. H. Su, S. Chakrabarti, and P. Bhattacharya, "Contribution of field-assisted tunneling emission to dark current in InAs/GaAs quantum dot infrared photodetectors," IEEE Photon. Technol. Lett. 16, $867 \quad$ (2004) [doi:10.1109/LPT.2004.823690].

[54] T. N. Theis, P. M. Mooney, and S. L. Wright, "Electron localization by a metastable donor level in n-GaAs: A new mechanism limiting the free-carrier density," Phys. Rev.Lett. 60, 361-364 (1988) [doi:10.1103/PhysRevLett.60.361].

[55] T. N. Morgan, "The DX centre," Semicond. Sci. Technol. 6, B23-B26 (1991) [doi: 10.1088/0268-1242/6/10B/004].

[56] E. F. Schubert, Doping in III-V Semiconductors, Cambridge University Press, Cambridge, United Kingdom (1993).

[57] M. Asche and O. G. Sarbey, "DX-center formation in planar-doped GaAs:Si in strong electric fields," J. Exp. Theor. Phys. 99, 574-584 (2004) [doi:10.1134/1.1809687].

[58] G. Vincent, D. Bois, and A. Chantre, "Photoelectric memory effect in GaAs," $J$. Appl. Phys. 53, 3643 (1982) [doi:10.1063/1.331147].

[59] J. C. Bourgoin and T. Neffati, "Detection of the metastable state of the EL2 defect in GaAs," J. Appl. Phys. 82, 4124 (1997) [doi:10.1063/1.365724].

[60] Z. Zhao, C. Yi, K. R. Lantz, and A. D. Stiff-Roberts, "Effect of donor-complexdefect-induced dipole field on InAs/GaAs quantum dot infrared photodetector activation energy," Appl. Phys. Lett. 90, 233511 (2007) [doi:10.1063/1.2747199].

[61] Z. Y. Zhao, C. Yi, A. D. Stiff-Roberts, A. J. Hoffman, D. Wasserman, and C. Gmachl, "Probing dopant incorporation in InAs/GaAs QDIPs by polarizationdependent Fourier transform infrared spectroscopy," Infrared Phys. Technol. 51, 131-135 (2007) [doi:10.1016/j.infrared.2007.04.002].

[62] Z. Zhao, C. Yi, A. D. Stiff-Roberts, A. J. Hoffman, D. Wasserman, and C. Gmachl, "DX-like centers in InAs/GaAs QDIPs observed by polarization-dependent Fourier transform infrared spectroscopy," J. Vac. Sci. Technol. B 25, 1108-1112 (2007) [doi:10.1116/1.2484803].

[63] http://www.corebyindigo.com/photon/photon320.cfm.

[64] http://www.cedip-infrared.com/infraredthermography/produit_detail.php?id=C9803 .

[65] http://www.infraredresearchcameras.com/cameras/camera/1096/. 
[66] S. Sauvage, P. Boucaud, F. H. Julien, J.-M. Gérard, and J.-Y. Marzin, "Infrared spectroscopy of intraband transitions in self-organized InAs/GaAs quantum dots," $J$. Appl. Phys. 82, 3396-3401 (1997) [doi:10.1063/1.365654].

[67] J. Phillips, K. Kamath, X. Zhou, N. Chervela, and P. Bhattacharya, "Photoluminescence and far-infrared absorption in Si-doped self-organized InAs quantum dots," Appl. Phys. Lett. 71, 2079-2081 (1997) [doi:10.1063/1.119347].

[68] K. W. Berryman, S. A. Lyon, and M. Segev, "Electronic structure and optical behavior of self-assembled InAs quantum dots," J. Vac. Sci. Technol. B 15, 10451050 (1997) [doi:10.1116/1.589390].

[69] Q. D. Zhuang, J. M. Li, H. X. Li, Y. P. Zeng, and L. Pan, "Intraband absorption in the 8-12 $\mu \mathrm{m}$ band from Si-doped vertically aligned InGaAs/GaAs quantum-dot superlattice," Appl. Phys. Lett. 73, 3706-3708 (1998) [doi:10.1063/1.122870].

[70] L. Chu, A. Zrenner, G. Böhm, and G. Abstreiter, "Normal-incident intersubband photocurrent spectroscopy on InAs/GaAs quantum dots," Appl. Phys. Lett. 75, 35993602 (1999) [doi:10.1063/1.125400].

[71] I. Mukhametzhanov, Z. H. Chen, O. Baklenov, E. T. Kim, and A. Madhukar, "Optical and photocurrent spectroscopy studies of inter- and intra-band transitions in size-tailored InAs/GaAs quantum dots," phys. stat. sol. (b) 224, 697-702 (2001) [doi:10.1002/(SICI)1521-3951(200104)224:3<697::AID-PSSB697>3.0.CO;2-M].

[72] Z. Chen, E.-T. Kim, and A. Madhukar, "Normal-incidence voltage-tunable middleand long-wavelength infrared photoresponse in self-assembled InAs quantum dots," Appl. Phys. Lett. 80, 2490-2492 (2002) [doi:10.1063/1.1467974].

[73] M. Schramboeck, A. M. Andrews, P. Klang, W. Schrenk, G. Hesser, F. Schaffler, and G. Strasser, "InAs/AlGaAs QDs for intersubband devices," Superlattice Microstruct. 44, 411-415 (2008) [doi:10.1016/j.spmi.2007.10.010].

[74] M. Schramboeck, A. M. Andrews, T. Roch, W. Schrenk, and G. Strasser, "In-based quantum dots on $\mathrm{Al}_{\mathrm{x}} \mathrm{Ga}_{1-\mathrm{x}} \mathrm{As}$ surfaces," Microelectron. Eng. 84, 1443 (2007) [doi:10.1016/j.mee.2007.01.228].

[75] W. Zhang, H.-C. Lim, M. Taguchi, S. Tsao, J. Szafraniec, B. Movaghar, M. Razeghi, and M. Tidrow, "High performance InAs quantum dot infrared photodetectors (QDIP) on InP by MOCVD," Proc. SPIE 5732, 326-333 (2005) [doi:10.1117/12.597139].

[76] A. Weber, O. Gauthier-Lafaye, F. H. Julien, J. Brault, M. Gendry, Y. Désieres, and T. Benyattou, "Strong normal-incidence infrared absorption in self-organized InAs/InAlAs quantum dots grown on $\operatorname{InP}(001), "$ Appl. Phys. Lett. 74, 413-415 (1999) [doi:10.1063/1.123045].

[77] S. Kim, H. Mohseni, M. Erdtmann, E. Michel, C. Jelen, and M. Razeghi, "Growth and characterization of InGaAs/InGaP quantum dots for mid-infrared photoconductive detector," Appl. Phys. Lett. 73, 963-965 (1998) [doi:10.1063/1.122053].

[78] J. Szafraniec, S. Tsao, W. Zhang, H. Lim, M. Taguchi, A. A. Quivy, B. Movaghar, and M. Razeghi, "High-detectivity quantum-dot infrared photodetectors grown by metalorganic chemical-vapor deposition," Appl. Phys. Lett. 88, 121102 (2006) [doi:10.1063/1.2188056].

[79] J. L. Liu, W. G. Wu, A. Balandin, G. L. Jin, and K. L. Wang, "Intersubband absorption in boron-doped multiple Ge quantum dots," Appl. Phys. Lett. 74, 185-187 (1999) [doi:10.1063/1.123287].

[80] P. Boucaud, V. L. Thanh, S. Sauvage, D. DéBarre, and D. Bouchier, "Intraband absorption in Ge/Si self-assembled quantum dots," Appl. Phys. Lett. 74. 401-403 (1999) [doi:10.1063/1.123083]. 
[81] J. L. Liu, W. G. Wu, A. Balandin, G. Jin, Y. H. Luo, S. G. Thomas, Y. Lu, and K. L. Wang, "Observation of inter-sub-level transitions in modulation-doped Ge quantum dots," Appl. Phys. Lett. 75, 1745-1747 (1999) [doi:10.1063/1.124806].

[82] A. I. Yakimov, A. V. Dvurechenskii, Y. Y. Proskuryakov, A. I. Nikiforov, O. P. Pchelyakov, S. A. Teys, and A. K. Gutakovskii, "Normal-incidence infrared photoconductivity in Si p-i-n diode with embedded Ge self-assembled quantum dot," Appl. Phys. Lett. 75, 1413-1415 (1999) [doi:10.1063/1.124710].

[83] C. Miesner, O. Röthig, K. Brunner, and G. Abstreiter, "Mid-infrared photocurrent measurements on self-assembled Ge dots in Si," Physica E 7, 146-150 (2000) [doi:10.1016/S1386-9477(99)00268-4].

[84] A. I. Yakimov, A. V. Dvurechenskii, A. I. Nikiforov, and Y. Y. Proskuryakov, "Interlevel Ge/Si quantum dot infrared photodetector," J. Appl. Phys. 89, 5676-5681 (2001) [doi:10.1063/1.1346651].

[85] C.-H. Lin, C.-Y. Yu, P.-S. Kuo, C.-C. Chang, T.-H. Guo, and C. W. Liu, "Deltadoped MOS Ge/Si quantum dot/well infrared photodetector," Thin Solid Films 508, 389-392 (2006) [doi:10.1016/j.tsf.2005.06.109].

[86] S. Krishna, "Quantum dots-in-a-well infrared photodetectors," J. Phys. D: Appl. Phys. 38, 2142-2150 (2005) [doi:10.1088/0022-3727/38/13/010].

[87] E.-T. Kim, Z. Chen, and A. Madhukar, "Tailoring detection bands of InAs quantumdot infrared photodetectors using InxGa1-xAs strain-relieving quantum wells," Appl. Phys. Lett. 79, 3341-3343 (2001) [doi:10.1063/1.1417513].

[88] S. Raghavan, P. Rotella, A. Stintz, B. Fuchs, S. Krishna, C. Morath, D. A. Cardimona, and S. W. Kennerly, "High-responsivity, normal-incidence long-wave infrared $(\lambda=7.2 \mu \mathrm{m}) \mathrm{InAs} / \mathrm{In}_{0.15} \mathrm{Ga}_{0.85} \mathrm{As}$ dots-in-a-well detector," Appl. Phys. Lett. 81, 1369-1371 (2002) [doi:10.1063/1.1498009].

[89] X. H. Su, S. Chakrabarti, A. D. Stiff-Roberts, J. Singh, and P. Bhattacharya, "Quantum dot infrared photodetector design based on double-barrier resonant tunneling," Electron. Lett. 40, 1082-1083 (2004) [doi:10.1049/el:20045206].

[90] P. Bhattacharya, X. H. Su, S. Chakrabarti, G. Ariyawansa, and A. G. U. Perera, "Characteristics of a tunneling quantum dot infrared photodetector operating at room temperature," Appl. Phys. Lett. 86, 191106 (2005) [doi:10.1063/1.1923766].

[91] A. G. U. Perera, G. Ariyawansa, V. M. Apalkov, S. G. Matsik, X. H. Su, S. Chakrabarti, and P. Bhattacharya, "Wavelength and polarization selective multi-band tunneling quantum dot detectors," Opto-electron. Rev. 15, 223-228 (2007) [doi:10.2478/s11772-007-0024-6].

[92] G. Ariyawansa, A. G. U. Perera, X. H. Su, S. Chakrabarti, and P. Bhattacharya, "Multi-color tunneling quantum dot infrared photodetectors operating at room temperature," Infrared Phys. Technol. 50, 156-161 (2007) [doi:10.1016/j.infrared.2006.10.001].

[93] P. Bhattacharya, X. H. Su, G. Ariyawansa, and A. G. U. Perera, "High-temperature tunneling quantum-dot intersublevel detectors for mid-infrared to terahertz frequencies," Proc. IEEE 95, 1828-1837 (2007) [doi:10.1109/JPROC.2007.900968].

[94] X. H. Su, J. Yang, P. Bhattacharya, G. Ariyawansa, and A. G. U. Perera, "Terahertz detection with tunneling quantum dot intersublevel photodetector," Appl. Phys. Lett. 89, 031117 (2006) [doi:10.1063/1.2233808].

[95] A. V. Barve, S. Y. Shah, J. Shao, T. E. Vandervelde, R. V. Shenoi, W. Y. Jang, and S. Krishna, "Reduction in dark current using resonant tunneling barriers in quantum dots-in-a-well long wavelength infrared photodetector," Appl. Phys. Lett. 93, 131115 (2008) [doi:10.1063/1.2996410].

[96] C. B. Murray, S. Sun, W. Gaschler, H. Doyle, T. A. Betley, and C. R. Kagan, "Colloidal synthesis of nanocrystals and nanocrystal superlattices," IBM J. Res. Dev. 45, 47-56 (2001) 
[97] N. C. Greenham, X. Peng, and A. P. Alivisatos, "Charge separation and transport in conjugated polymer/cadmium selenide nanocrystal composites studied by photoluminesence quenching and photoconductivity," Syn. Met. 84, 545-546 (1997) [doi:10.1016/S0379-6779(97)80852-1].

[98] D. S. Ginger and N. C. Greenham, "Photoinduced electron transfer from conjugated polymers to CdSe nanocrystals," Phys. Rev. B 59, $10622-10629$ (1999) [doi:10.1103/PhysRevB.59.10622].

[99] C. A. Leatherdale, C. R. Kagan, N. Y. Morgan, S. A. Empedocles, M. A. Kastner, and M. G. Bawendi, "Photoconductivity in CdSe quantum dot solids," Phys. Rev. B 62, 2669-2680 (2000) [doi:10.1103/PhysRevB.62.2669].

[100] D. C. Oertel, M. G. Bawendi, A. C. Arango, and V. Bulovic, "Photodetectors based on treated CdSe quantum-dot films," Appl. Phys. Lett. 87, 213505(2005) [doi:10.1063/1.2136227].

[101] S. A. McDonald, P. W. Cyr, L. Levina, and E. H. Sargent, "Photoconductivity from $\mathrm{PbS}$-nanocrystal/semiconducting polymer composites for solution-processible, quantum-size tunable infrared photodetectors," Appl. Phys. Lett. 85, 2089-2091 (2004) [doi:10.1063/1.1792380].

[102] D. Qi, M. Fischbein, M. Drndic, and S. Selmic, "Efficient polymer-nanocrystal quantum-dot photodetectors," Appl. Phys. Lett. 86, $093103 \quad$ (2005) [doi:10.1063/1.1872216].

[103] K. R. Choudhury, Y. Sahoo, T. Y. Ohulchanskyy, and P. N. Prasad, "Efficient photoconductive devices at infrared wavelengths using quantum dot-polymer nanocomposites," Appl. Phys. Lett. 87, 073110 (2005) [doi:10.1063/1.2011768].

[104] G. Konstantatos, I. Howard, A. Fischer, S. Hoogland, J. Clifford, E. Klem, L. Levina, and E. H. Sargent, "Ultrasensitive solution-cast quantum dot photodetectors," Nature 442, 180-183 (2006) [doi:10.1038/nature04855].

Adrienne D. Stiff-Roberts is an Assistant Professor of Electrical and Computer Engineering at Duke University. Dr. Stiff-Roberts received both the B.S. degree in physics from Spelman College and the B.E.E. degree in electrical engineering from the Georgia Institute of Technology in 1999. She received the M.S.E. degree in electrical engineering and a the Ph.D. degree in applied physics in 2001 and 2004, respectively, from the University of Michigan, Ann Arbor. Dr. Stiff-Roberts is a member of Phi Beta Kappa, Sigma Pi Sigma, and IEEE. She is also a recipient of the NSF CAREER Award in 2006, the ONR Young Investigator Award in 2007, and the IEEE Early Career Award in Nanotechnology of the Nanotechnology Council in 2009. 